\title{
Ultrafast dynamics of ligand and substrate interaction in endothelial nitric oxide synthase under Soret excitation
}

\author{
Chih-Chang Hung, ${ }^{1}$ Atsushi Yabushita ${ }^{1,2,4^{*}}$ Takayoshi Kobayashi, ${ }^{1,3,4,5}$ Pei-Feng Chen, ${ }^{6}$ and Keng S. Liang ${ }^{6,7}$ \\ ${ }^{1}$ Department of Electrophysics, National Chiao Tung University, Hsinchu, Taiwan \\ ${ }^{2}$ Faculty of Engineering, Kanagawa University, 3-27-1 Rokkakubashi, Yokohama 221-8686, Japan \\ ${ }^{3}$ Department of Applied Physics and Chemistry and Institute for Laser Science, The University of \\ Electrocommunications, Tokyo, Japan \\ ${ }^{4}$ CREST, JST, Saitama, Japan \\ ${ }^{5}$ Institute of Laser Engineering, Osaka University \\ ${ }^{6}$ Institute of Physics, Academia Sinica, Taipei, Taiwan \\ ${ }^{7}$ Institute of Physics, National Chiao Tung University, Hsinchu, Taiwan
}

AUTHOR EMAIL ADDRESS: YABUSHITA@MAIL.NCTU.EDU.TW

RECEIVED DATE (to be automatically inserted after your manuscript is accepted if required according to the journal that you are submitting your paper to)

\section{CORRESPONDING AUTHOR FOOTNOTE.}

*To whom correspondence should be addressed: Department of Electrophysics, National Chiao-Tung University, Hsinchu 300, Taiwan. Telephone: 88635712121 ext56197. Fax: 8863 5725230. E-mail: yabushita@mail.nctu.edu.tw. 
ABSTRACT. Ultrafast transient absorption spectroscopy of endothelial NOS oxygenase domain (eNOS-oxy) was performed to study dynamics of ligand or substrate interaction under Soret band excitation. Photo-excitation dissociates imidazole ligand in less than $300 \mathrm{fs}$, then followed by vibrational cooling and recombination within 2 ps. Such impulsive bond breaking and late rebinding generate proteinquakes, which relaxes in several tens of picoseconds. The photo excited dynamics of eNOS-oxy with ${ }_{L}$-arginine substrate mainly occurs at the local site of heme, including ultrafast internal conversion within $400 \mathrm{fs,} \mathrm{vibrational} \mathrm{cooling,} \mathrm{charge} \mathrm{transfer,} \mathrm{and} \mathrm{complete} \mathrm{ground-state} \mathrm{recovery} \mathrm{within} 1.4$ ps. The eNOS-oxy without additive is partially bound with water molecule, thus its photoexcited dynamics also shows ligand dissociation in less than $800 \mathrm{fs}$. Then it followed by vibrational cooling coupled with charge transfer in $4.8 \mathrm{ps}$, and recombination of ligand to distal side of heme in $12 \mathrm{ps.}$

KEYWORDS. Ultrafast spectroscopy, Nitric Oxide Synthase, Photo-dissociation, Ligand rebinding

\section{Graphical Abstract}

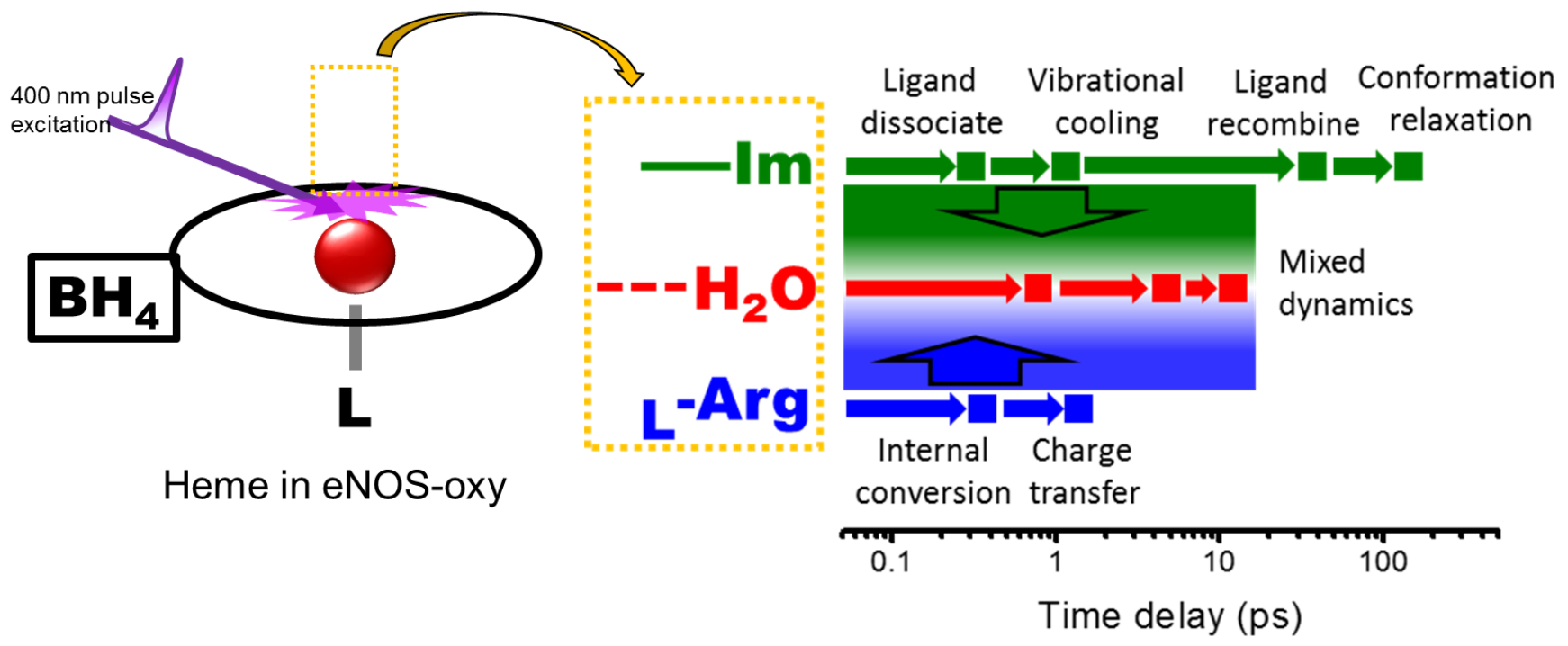




\section{MANUSCRIPT TEXT}

\section{Introduction}

Nitric oxide (NO) is a key signaling molecule for vasodilation and neurotransmission at low concentrations and a defensive cytotoxin at higher concentrations ${ }^{1,2}$. In mammals, NO is biosynthesized with $_{\mathrm{L}}$-citrulline from ${ }_{\mathrm{L}}$-arginine by three isoforms (neuronal, inducible, and endothelial) of nitric oxide synthases (NOS) called as nNOS, iNOS, and eNOS, respectively ${ }^{3}$. The NO produced by eNOS in the endothelial cells signalizes blood pressure control, one produced by nNOS in the brain is for nerve signal transduction ${ }^{4}$, and one produced by iNOS in macrophages triggers immune defense ${ }^{5}$. All of these three isoforms share similar biochemical composition and enzymatic characteristics. They are all homodimeric enzymes including heme, FAD, FMN and tetrahydrobiopterin $\left(\mathrm{BH}_{4}\right)^{6,7,8,9,10}$. Comparison of sequence homology of the isoforms suggested that these isozymes possess a bidomain structure similar to that of cytochrome $\mathrm{P} 450_{\mathrm{BM}-3}$ being catalytically self-sufficient ${ }^{11}$. In the bidomain structure, the C-terminal half (reductase domain) contains the NADPH, FAD, and FMN recognition sites. The other half, which is called the $\mathrm{N}$-terminal half (oxygenase domain), contains heme ${ }^{12}, \mathrm{BH}_{4}$, and $\mathrm{L}_{\text {-arginine }}$ binding $\operatorname{site}^{13}$. $\mathrm{A} \mathrm{Ca}^{2+} / \mathrm{CaM}$ binding site located in the middle of the two domains ${ }^{14}$ is considered to be important in facilitating electron transfer from the reductase domain to the oxygenase domain ${ }^{15}$.

To control the density of NO via pharmacological intervention against individual isoforms, systematical and detailed understanding of the NOS reaction mechanism is essentially important. In the NOS reaction mechanism, the oxygenase domain of NOS especially plays the key role as follows. The 
oxygenase domain contains the thiolate ligand in proximal side of heme, the distal heme pocket as ligand or substrate binding regions. The catalytic process of NO production occurs in the heme active site of the NOS oxygenase domain.

Because of the biological importance of the NOS, NOS and NOS-like proteins have been widely studied in various fields. Chen et al. ${ }^{16}$ has shown the spectral evidence of domain structure and catalytic activity of human eNOS from two separate domains, i.e. oxygenase domain and reductase domain. Gerber et $a l .{ }^{17}$ have demonstrated that the heme ligand (imidazole) or substrate (L-arginine) could induce spin state change showing a clear Soret peak shift of heme in wild-type eNOS. Ligand-protein interaction in NOS has been systematically studied by the resonance Raman scattering spectroscopy ${ }^{18}$ showing that the electronic properties and the structure of the proximal iron-cysteine bond of heme in NOS system are important to assess its contribution to the catalytic function. The rapid kinetics of the intramolecular electron transfer between the FMN and heme domains in NOS system was directly determined using laser flash photolysis of $\mathrm{CO}$ dissociation and stopped flow system in comparative studies with millisecond time-resolution ${ }^{19,20}$. Previously, the ultrafast dynamics of the NOS proteins were partially studied with picosecond time-resolution ${ }^{21,22}$, which is still not enough to fully understand the mechanism of the NOS protein dynamics. Especially, elucidation of the ultrafast heme dynamics in the oxygenase domain is thought to be mostly important because the catalytic process of NO production occurs in the heme active site of the oxygenase domain. Previous studies show that the catalytic center of NOS is affected by heme ligands and substrates to modify the enzymatic activity ${ }^{23}$. 
In our presented work, the ultrafast dynamics of eNOS oxygenase domain (eNOS-oxy) have been studied by measuring transient absorption (TA) with femtosecond time resolution and estimated effect of ligand or substrate coordination to heme.

\section{Experimental section}

\subsection{Experimental setup of TA spectroscopy}

The light source of TA spectroscopy was the Ti:sapphire regenerative amplifier (Legend, Coherent Inc.), which generates NIR pulse with central wavelength of $800 \mathrm{~nm}$, repetition rate of $5 \mathrm{kHz}$, spectral bandwidth of $30 \mathrm{~nm}$, and pulse duration of 35 fs. The NIR pulse was frequency-doubled by a 0.5-mm-thick $\beta-\mathrm{BaB}_{2} \mathrm{O}_{4}$ crystal to generate UV pulse with bandwidth of $20 \mathrm{~nm}$ around $400 \mathrm{~nm}$. The remnant NIR pulse was cut by a glass filter, which passes light with wavelength shorter than $750 \mathrm{~nm}$. A beam sampler was inserted to separate the UV pulse into two copies with the power ratio of about 9:1. The UV pulse transmitted through the beam sampler with higher intensity was used as pump pulse. The UV pulse reflected on the beam sampler with lower intensity was served as probe pulse. The pump pulse was modulated by chopper rotating at $2.5 \mathrm{kHz}$ which is synchronized with the 5-kHz repetition rate of the regenerative amplifier. To suppress coherent artifact in the TA signal, the polarizations of pump pulses and probe pulses were set orthogonal to each other. Both pulses were focused on the sample by an off-axis parabolic mirror with focal length of $100 \mathrm{~mm}$. The transmitted probe pulse through the sample was coupled into an optical fiber and guided into a polychromator to be dispersed 
with grating $(150 \mathrm{~g} / \mathrm{mm})$. The dispersed spectrum of the probe pulse was detected by a fast scan-rate

CCD with 256 pixel for every laser pulse. The timing of the data collection in the CCD was synchronized with the pulsed probe light source at $5-\mathrm{kHz}$ repetition rate. The TA spectra can be calculated at every $0.4 \mathrm{~ms}$ for every two probe pulses (with pump and without pump). To enhance the signal to noise ratio, we have accumulated the TA spectra for $0.5 \mathrm{~s}$ (2500 shots) at each time delay and averaged the TA time trace scanning the time delay for four times. The temporal resolution in the pump-probe measurement system was estimated to be $100 \mathrm{fs}$. The pump and probe pulse energies were $45 \mathrm{~nJ}$ and $5 \mathrm{~nJ}$, respectively. To suppress the effect of material chirp, the TA spectroscopy of the liquid sample was performed storing the sample solution in a thin glass cell with 1-mm optical path length (6210-12501, GL Sciences Inc.). All measurements were performed at room temperature of $295 \mathrm{~K}$.

\subsection{Preparation of eNOS oxygenase domain}

2.2.1 Expression and Purification of eNOS oxygenase domain-The Sf21 cells were cotransfected with Eoxyhis1392 plasmid and linearized BaculoGold baculovirus DNA (BD Biosciences/Pharmingen) to generate recombinant viruses. Due to low heme biosynthetic capability of Sf21 cells, supplemental heme chloride $(4 \mu \mathrm{g} / \mathrm{ml})$ was added into the culture medium $18 \mathrm{~h}$ postinfection to enrich heme content of the expressed eNOS oxygenase domain. Cells were harvested at $60 \mathrm{~h}$ postinfection, suspended in 5 volumes of buffer A containing $25 \mathrm{mM}$ Tris-HCl, $\mathrm{pH}$ 8.0, 0.5 M NaCl, $1 \mu \mathrm{M}$ leupeptin, $1 \mu \mathrm{M}$ antipain, 1 $\mu \mathrm{M}$ pepstatin A, $1 \mathrm{mM}$ phenylmethylsulfonyl fluoride and $10 \%$ glycerol. The cell suspension was 
sonicated three times for $20 \mathrm{~s}$ each and centrifuged twice at $30,000 \mathrm{x} \mathrm{g}$ for $20 \mathrm{~min}$ at $4{ }^{\circ} \mathrm{C}$. The supernatant was applied to a 5-ml column of Ni-NTA-agarose equilibrated with buffer A. The column was washed with buffer A, then with buffer A plus $5 \mathrm{mM}$ imidazole. The column was eluted with buffer containing $25 \mathrm{mM}$ Tris-HCl, pH 8.0, 0.5 M NaCl, 10\%glycerol plus $100 \mathrm{mM}$ imidazole. The eluate was concentrated by using Centriprep-30 (Amicon) and dialyzed against buffer containing $25 \mathrm{mM}$ Tris- $\mathrm{HCl}$, pH7.5, $100 \mathrm{mM} \mathrm{NaCl}, 0.1 \mathrm{mM}$ DTT, and 10\% glycerol. The concentration of eNOS oxygenase domain was either determined by Bradford method or estimated spectroscopically using $1 \mathrm{~A}_{280 \mathrm{~nm}}=0.63 \mathrm{mg} / \mathrm{ml}$. The purified enzyme preparations were stored at $-80{ }^{\circ} \mathrm{C}$.

2.2.2 Sample Measurement — Imidazole and ${ }_{\mathrm{L}}$-arginine were purchased from Sigma-Aldrich (St. Louis, MO) and used without further purifications. Imidazole and ${ }_{\mathrm{L}}$-arginine were dissolved in $25 \mathrm{mM}$ Tris-HCl, pH7.5, $100 \mathrm{mM} \mathrm{NaCl}, 0.1 \mathrm{mM}$ DTT, and $10 \%$ glycerol. The solution was filtered through Nalgene $0.45 \mu \mathrm{M}$ PES filter from Thermo Scientific (Waltham, MA). The final concentration of the eNOS-oxy solution was determined to be $\approx 10 \mu \mathrm{M}$ from the UV/Vis absorption spectrum in the Soret region. Imidazole and $\mathrm{L}_{\mathrm{L}}$-arginine were added to the solution to form corresponding complexes at final concentrations of $1 \mathrm{mM}$ and $100 \mu \mathrm{M}$, respectively.

\section{Results and Discussion}

\subsection{Stationary absorption spectra}


Figure 1 shows stationary absorption spectra in Soret band region of eNOS-oxy, eNOS-oxy with

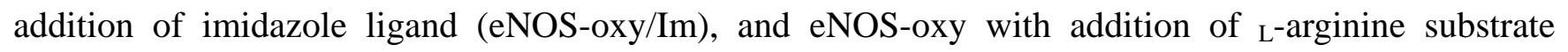
$(\mathrm{eNOS}-\mathrm{oxy} / \mathrm{L}-\mathrm{Arg})$

The Soret band associated with the $\pi$ to $\pi^{*}$ electronic transition of the porphyrin macrocycle is sensitive to the oxidation, coordination and spin states of the heme iron in heme protein. Ferric derivative of NOS adopts a six-coordinate low-spin (LS) state configuration, possibly coodinating a solvent water molecule at the distal binding site of the heme. Addition of $\mathrm{BH}_{4}$ destabilizes the putative heme-bound water molecule through steric interference and structure changes to the protein matrix.

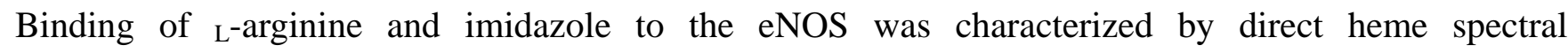
perturbation ${ }^{25}$. When both ${ }_{\mathrm{L}}$-arginine and $\mathrm{BH}_{4}$ are present with eNOS, the Soret transition peak futher shifts to $395 \mathrm{~nm}$, indicating a full conversion to the five-coordinated high-spin (HS) state. When imidazole is added to the eNOS, imidazole acts as a genuine heme ligand coordinating to distal side of heme iron in eNOS directly and thus the Soret transition shifts to $430 \mathrm{~nm}$. The red shift represents that heme in eNOS has the six-coordinated LS. When neither of ${ }_{\mathrm{L}}$-arginine and imidazole is added to eNOS, the Soret peak shifts to $\sim 400 \mathrm{~nm}$ indicating co-existence of the six-coordinated LS and the five coordinated HS.

Our observation is consistent with the results of absorption spectroscopy of rat brain NOS and eNOS added with imidazole or $\mathrm{L}_{\text {-arginine }}{ }^{24,25}$. According to experimental work by Maes et al. ${ }^{26}$, the Soret peak of $\mathrm{Fe}(\mathrm{III})$ and $\mathrm{Fe}(\mathrm{II})$ of Nitrophorin 4 (NP4) bound with water molecule appears at $404 \mathrm{~nm}$ 
and $421 \mathrm{~nm}$, respectively. The Soret peak of Fe(III) and Fe(II) of tetraphenylphorphine tetrasulfonic acid (TPPS) locates at $393 \mathrm{~nm}$ and $421 \mathrm{~nm}$, respectively ${ }^{27}$. Thus, the observed broad Soret peak of eNOS-oxy is attributed to the electronic configuration mixing in heme between ferric five-coordination HS and ferous six-coordination LS.

\subsection{Transient absorption time traces and analysis method}

Figures 2, 3, and 4 show the femtosecond spectra integrated TA traces of the three samples for eNOS-oxy/Im, eNOS-oxy/L-Arg, and eNOS-oxy, respectively. The TA traces were measured pumping at $405 \mathrm{~nm}$ and probing also at $405 \mathrm{~nm}$ with spectra bandwidth of $\sim 20 \mathrm{~nm}$. The meaured TA traces were fitted with triple-exponential decay function and the fitting results are summerized in Table 1.

The femtosecond TA traces show clear difference between the three samples. The TA signal of the eNOS-oxy and eNOS-oxy/Im is negative just after photo-excitation gradually increasing to have positive sign in a few picosecond. Meanwhile, the TA signal of eNOS-oxy/L-Arg exhibits only negative signal with ultrafast relaxation. Thus, the three samples have distinct excited-state dynamics and different protein responses. The following sections provide detail explantions about the difference observed in the ultrafast dynamics of these samples.

\subsection{Photoexcited dynamics of eNOS-oxy bound with imidazole ligand}

As shown in figure 2, the spectra integrated TA trace of eNOS-oxy/Im initially show negative 
signal of photo-bleaching within time delay $~ 0.3$ ps then followed by positive signal of induced absorption. The ultrafast change is caused by heme-ligand dissociation in eNOS-oxy/Im transforming heme structure from six to five coordination after photo-excitation. Just after the photo-excitation, heme in eNOS-oxy/Im has six coordination bound with imidazole. After the ultrafast heme-ligand dissociation, the sign of TA signal changes to be positive reflecting the induced absorption of the newly formed five-fold coordinated heme. The observed time scale of photo-dissociation of ligand is consistent with that reported by Negrerie et al. ${ }^{28}$ at Q-band excitation and that by Wang et al. ${ }^{29}$ at the Soret band (B-band) excitation. The heme dynamics of cytochrome c pumped at the Soret band and probed in UV and visible region was reported by Zang et al. ${ }^{30}$, which indicates that ligand dissociates in less than 100 fs. Theoretical work by molecular dynamics (MD) ${ }^{31}$ shows that ligand dissociation occurs in $\sim 200 \mathrm{fs}$. The ultrafast photo-excitation was reported to break the heme-ligand bond in less than 300 fs, which perturbs protein structure significantly forming protein-quake $32,33,34$ at the local heme site. The quake spreads outward to fluctuate protein conformation.

The TA trace probed at $405 \mathrm{~nm}$ is plotted in Fig. 2, which shows that negative TA signal rapidly decays in 0.36 ps $(76.5 \%)$ by photo-dissociation of imidazole ligand from heme of eNOS-oxy/Im. Then, the positive TA signal relaxes in $1.2 \mathrm{ps}(18.3 \%)$ and $144 \mathrm{ps}(5.2 \%)$. The former time constant corresponds to the vibrational cooling. The latter one reflects heme-imidazole recombination and protein conformation change induced by the impulsive protein quake recovers in 144 ps. Thus, the ultrafast dynamics of eNOS-oxy/Im is summarized as follows. The photo-dissociation of 
heme-imidazole is the primary reaction breaking the bond in less than $0.36 \mathrm{ps}$, and forms the vibrational hot state of the five-fold coordinated heme. Then the cascaded vibrational cooling occurs within 2 ps, accompanying heme-imidazole ligand recombination and relaxation of the local heme site within 37.5 ps (see supporting information and Fig. S2). Finally, the collective structural relaxations take 144 ps to completely dissipate energy to the heme environment and have the conformation to be fully recovered.

\subsection{Photoexcited dynamics of eNOS-oxy domain with ${ }_{L}$-arginine substrate}

The TA trace of eNOS-oxy/L-Arg excited at $405 \mathrm{~nm}$ is shown in Fig. 3. At all of the probe wavelengths, we have observed negative TA signal for eNOS-oxy/L-Arg being different from that for eNOS-oxy/Im. Fitting result of the TA signal estimated two lifetimes of 0.35 and 1.4 ps. A water molecule is bound on distal side of heme in stationary condition of eNOS-oxy. Meanwhile, in the stationary condition of eNOS-oxy/L-Arg, ${ }_{\mathrm{L}}$-arginine kicks out the water molecule on distal side of heme to form five coordination. Thus, the observed ultrafast dynamics of the eNOS-oxy/L-Arg is not representing heme-ligand dissociation but the relaxation of the excited five-fold coordinated heme of eNOS-oxy. It should be emphasized here that the TA signal probed at $405 \mathrm{~nm}$ did not show relaxation later than 20 ps, which implies that slow conformation relaxation observed in >20ps for eNOS-oxy/Im does not occur in eNOS-oxy/L-Arg.

The ultrafast decay in 0.35 ps corresponds to the relaxation of population distributed in the higher vibrational levels to the lower levels in the Soret band electronic state via vibrational relaxation. The 
results of time resolved resonance Raman scattering show that heme charge-transfer band is vibronically coupled to the Soret band and indicated axial iron out-of-plane displacement is primarily responsible for the structure-dynamics correlations observed in myoglobin ${ }^{35}$. Moreover, the longer time constant of 1.4 ps in TA signal could be related to the porphyrin ring to iron charge transfer. It is similar with photophysical pathway arises from porphyrin ring-to-iron back charge transfer to produce a porphyrin ground state configuration a nonequilibrium iron d-orbital population ${ }^{36}$.

\subsection{Photoexcited dynamics of eNOS-oxy}

As shown in Fig. 4, the TA signal of eNOS-oxy shows initial negative signal decaying in $\sim 2.5 \mathrm{ps}$ followed by gradual change to be positive. This trend is similar with eNOS-oxy/Im. The decay lifetime of the negative signal is similar to that of eNOS-oxy $/ L-A r g$. Water molecule weakly is bound in distal side of heme in eNOS-oxy results in imperfect six coordination with low spin configuration. Thus, the dynamics of eNOS-oxy can be consisted of that of eNOS-oxy/L-Arg (five coordination) and that of eNOS-oxy/Im (six coordination). The negative TA signal of eNOS-oxy reflects photo-dissociation of water molecule, metal to porphyrin charge transfer, and virbational cooling, all of which have been estimated to occur earlier than $2.5 \mathrm{ps}$ in previous sections. Then, positive TA signal is thought to be due to water molecules rebinding to the distal side of heme occuring in a few tens of picoseconds.

The TA signal of eNOS-oxy was fitted to three exponential functions with the time constants as $0.79,4.76$, and $12.2 \mathrm{ps}$. It implies that the photo-excitation dissociate water molecule on distal side of eNOS-oxy with time constant of 0.79 ps. Then, the virbational cooling couple with charge transfer have 
occurred in $4.76 \mathrm{ps}$, and the dissociated water moelcule rebinds to distal side of heme in $12.2 \mathrm{ps}$.

The photophysics of oxidized cytochrome (Cyt) c complex has been studied with femtosecond Raman and TA spectroscopy indicating that vibration relaxation of heme does occur in $\sim 7 \mathrm{ps}^{28}$. The results of Ursula et $a .^{37}$ suggest that photoexcitation of the ferric heme domain of direct oxygen sensor dissociates ligand of $\mathrm{H}_{2} \mathrm{O}$ and rebinds it in $\sim 20 \mathrm{ps}^{38,39}$. Femtosecond infrared (IR) spectroscopy of deoxy-hemoglobin and deoxy-myoglobin $(\mathrm{Mb})$ has shown that $\mathrm{D}_{2} \mathrm{O}$ molecule works as heat acceptor perspective with thermal relaxation in double exponential function with a fast component of $\sim 8$ ps and a slow component of $\sim 20$ ps. Femtosecond near-IR transient absorbance study of the band III of photoexcited $\mathrm{Mb}$ where the longest relaxation time constant of about 6 ps was obtained and interpreted as vibrational cooling of the electronically relaxed heme ${ }^{40}$. Previously study has shown that vibrational cooling proceeds in ferrous Cyt $c$ with relaxation time constants $\sim 3$ ps, while the lifetime of $\sim 6$ ps was attributed to ligand rebinding ${ }^{29}$. The ultrafast dynamics of eNOS-oxy obtained in the present work agrees with above mentioned previous works.

Referring the result obtained for eNOS-oxy/Im and eNOS-oxy/L-Arg, we have clarified the ultrafast dynamics of eNOS-oxy as following. The photo-dissociation of heme- $\mathrm{H}_{2} \mathrm{O}$ is the primary reaction breaking the binding in $0.79 \mathrm{ps}$. After ligand dissociation, five-fold coordinated heme is formed in vibrational excited state, which is cooled in 4.76 ps accompanying heme- $\mathrm{H}_{2} \mathrm{O}$ rebinding processes. The complete relaxation of the local heme site takes $12.2 \mathrm{ps}$. 


\section{Conclusion}

We have performed TA spectroscopy of eNOS-oxy, eNOS-oxy/Im, and eNOS-oxy/L-Arg pumping and probing at Soret band with femtosecond time-resolution. The experimental result has elucidate local heme dynamics and photo-induced conformation relaxation affected by ligand or substrate as follows.

When the eNOS-oxy/Im is photo-excited, heme-imidazole bond was dissociated in 300 fs to form proteinquake and followed by a series of vibrational cooling including local structure relaxation of porphyrin in heme with 2 ps. The proteinquake at the local heme site faded away recovering heme in few tens ps. Total recovery of conformation change took 144 ps. After photo-excitation of the eNOS-oxy $/ \mathrm{L}$-Arg, intramolecular and subsequent intermolecular vibrational redistributions occurred in less than 400 fs. The metal to porphyrin ring charger transfer was found to take 1.4 ps.

The eNOS-oxy in stationary state is mixed state consisted of five coordination and six coordination. The observed photo-induced dynamics of eNOS-oxy could be explained as mixture of the dynamics of eNOS/L-Arg (initially 5-coordination) and the dynamics of eNOS/Im (initially 6-coordination). We have demonstrated that the photo-excitation of eNOS-oxy leads the fast $(0.79 \mathrm{ps})$ photo-dissociation of water molecule ligand to create a non-equilibrium five coordinate species. The vibrational cooling coupled with charge transfer is estimated to occur in 4.76 ps accompanied with heme- $\mathrm{H}_{2} \mathrm{O}$ rebinding process. It is followed by heme relaxation with a time constant of $12.2 \mathrm{ps}$.

In conclusion, we have reported comprehensive investigation of the ultrafast dynamics for ligand and substrate interaction in heme of eNOS-oxy domain, which is expected to give key knowledge for 
future work on pharmacological intervention against individual isoforms of NOS contributing to human health.

Acknowledgement. This work was supported by ministry of science technology, Taiwan (MOST 104-2112-M-009-001-MY2) and JSPS KAKENHI Grant Number 16K17784. This work was also supported in part by Japan Science and Technology Agency (JST). 


\section{Figure Captions}

Fig. 1 Stationary absorption spectra of the three eNOS-oxy samples; eNOS-oxy (black curves), eNOS-oxy/Im (blue curves), and eNOS-oxy/L-Arg (brown curves).

Fig. 2 Transient absorption trace (blue solid curves) of eNOS-oxy/Im excited and probed both at $405 \mathrm{~nm}$.

The fitting curve (yellow dash curves) contains three components of negative signal with time constant $0.31 \mathrm{ps}$ (green dash curves), short-life positive signal with lifetime of $1.44 \mathrm{ps}$ (orange dash curves), long-life positive signal decaying in 144 ps (purple dash curves). The inset shows the estimated percentage of signal amplitude for each lifetime component.

Fig. 3 Transient absorption trace (brown solid curves) of eNOS-oxy/L-Arg pumped and probed both at $405 \mathrm{~nm}$. The fitting curve (yellow dash curves) contains two components plotted by green and orange dash curves representing negative signal decaying with time constant of 0.35 ps and 1.41 ps, respectively. The inset shows the percentage of signal amplitude of each lifetime component.

Fig. 4 Transient absorption trace (black solid curves) of eNOS-oxy domain excited and probed both at $405 \mathrm{~nm}$. The fitting curve (yellow dash curves) consists of three components of negative signal with lifetime of 0.79 ps (green dash curves), negative signal decaying within 4.76 ps (orange dash curves), and positive signal with time constant of $12.2 \mathrm{ps}$ (purple dash curves). The inset shows the percentage of signal amplitude of each lifetime component. 
Fig. 1

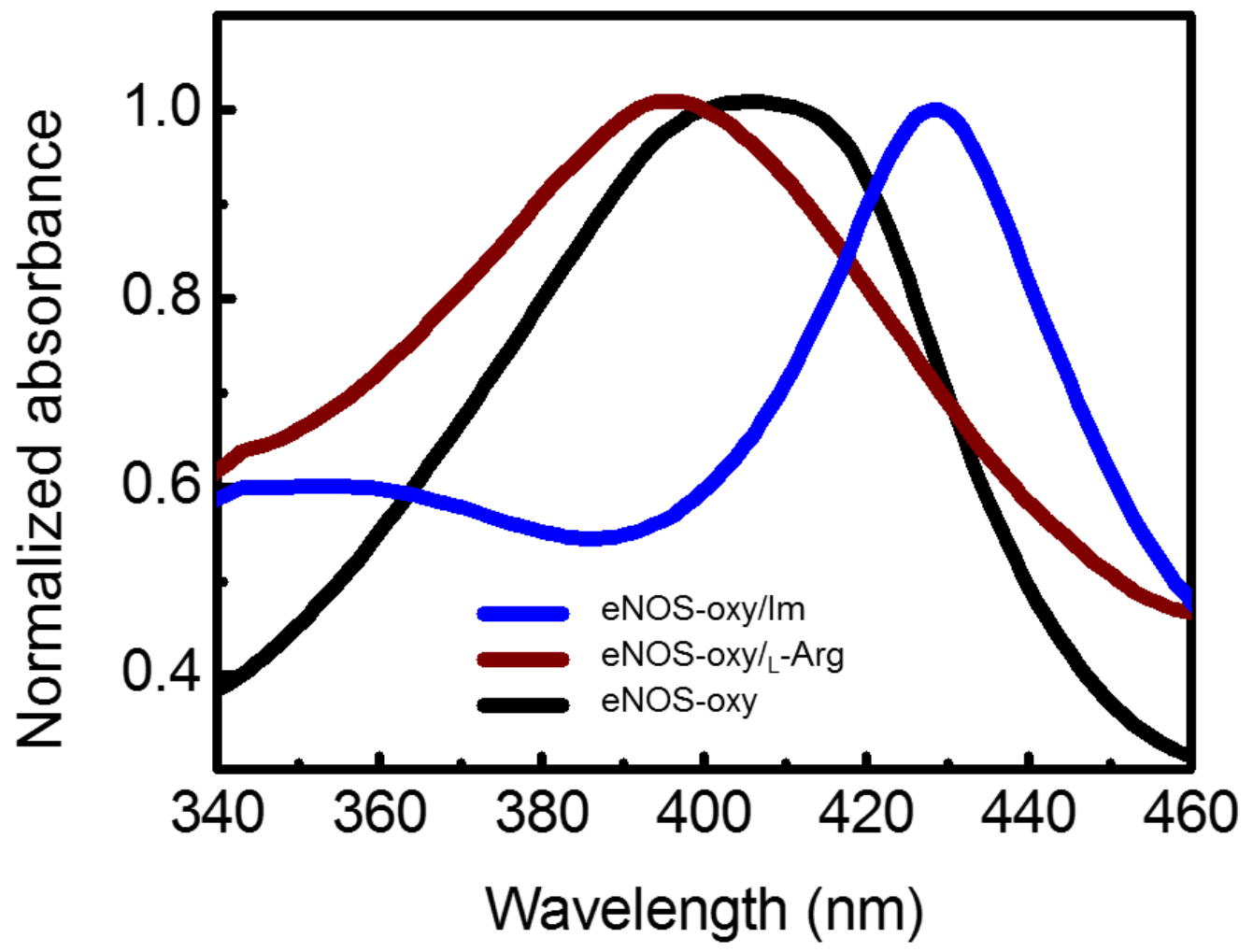

Fig. 2

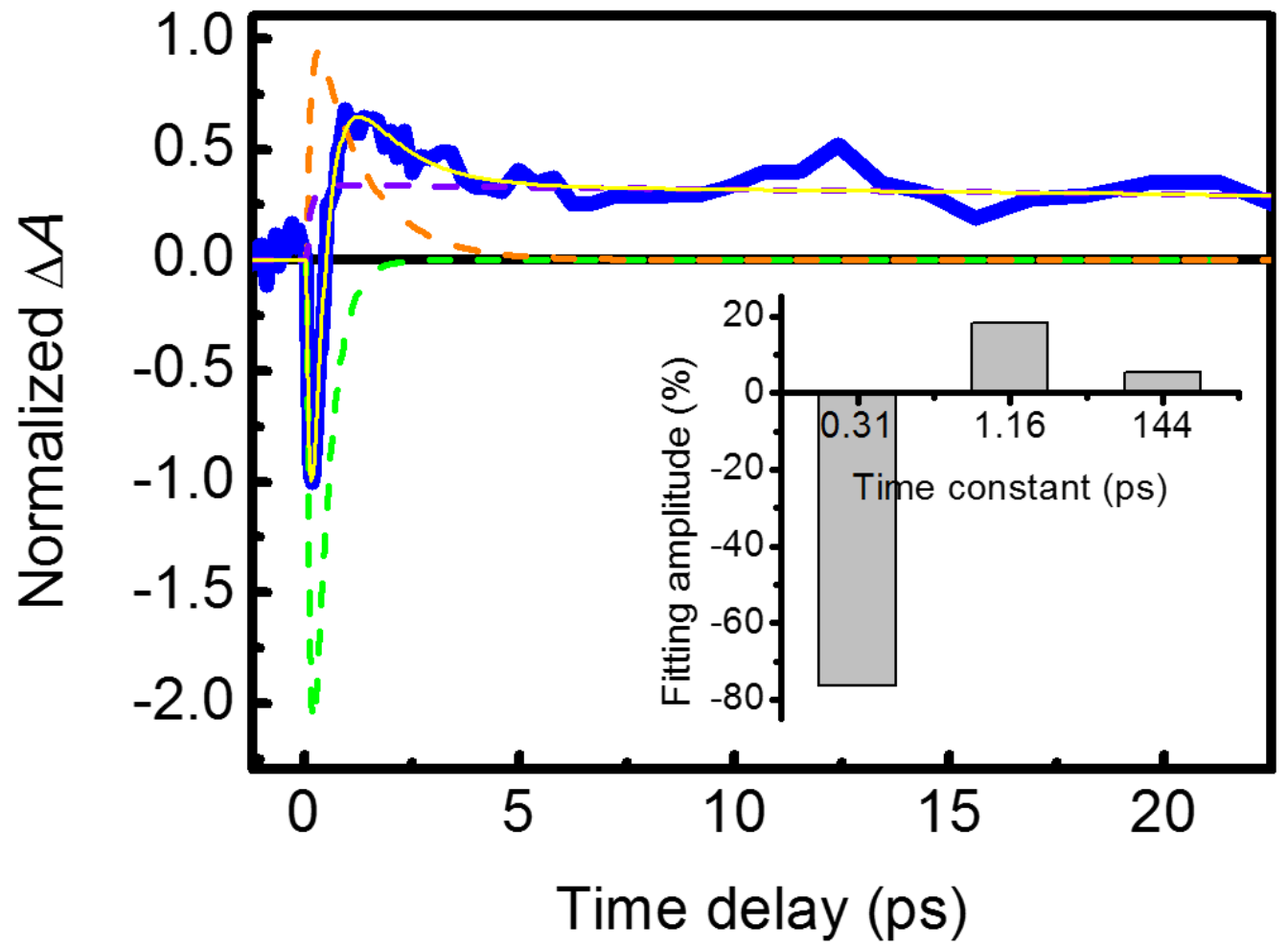


Fig. 3

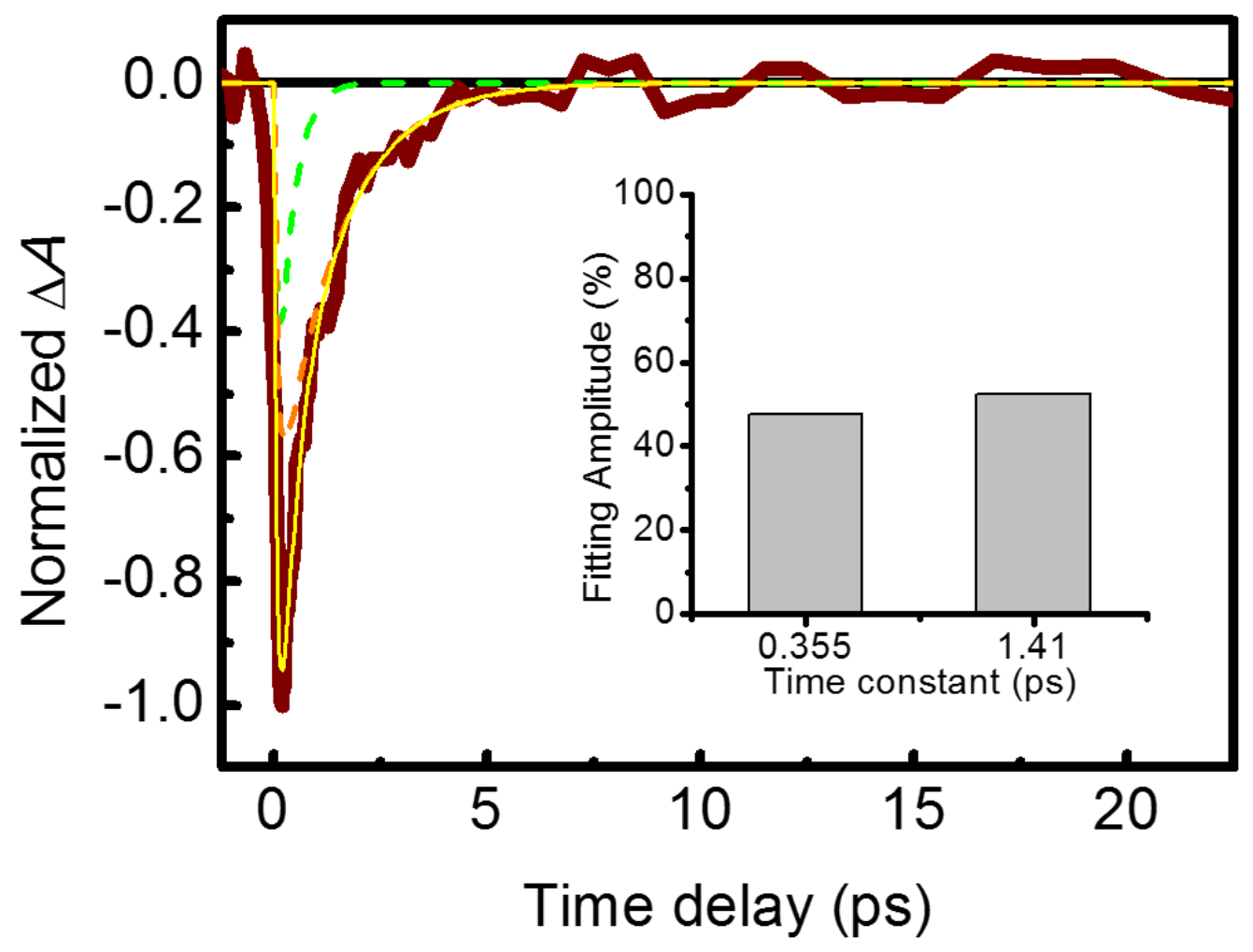

Fig. 4

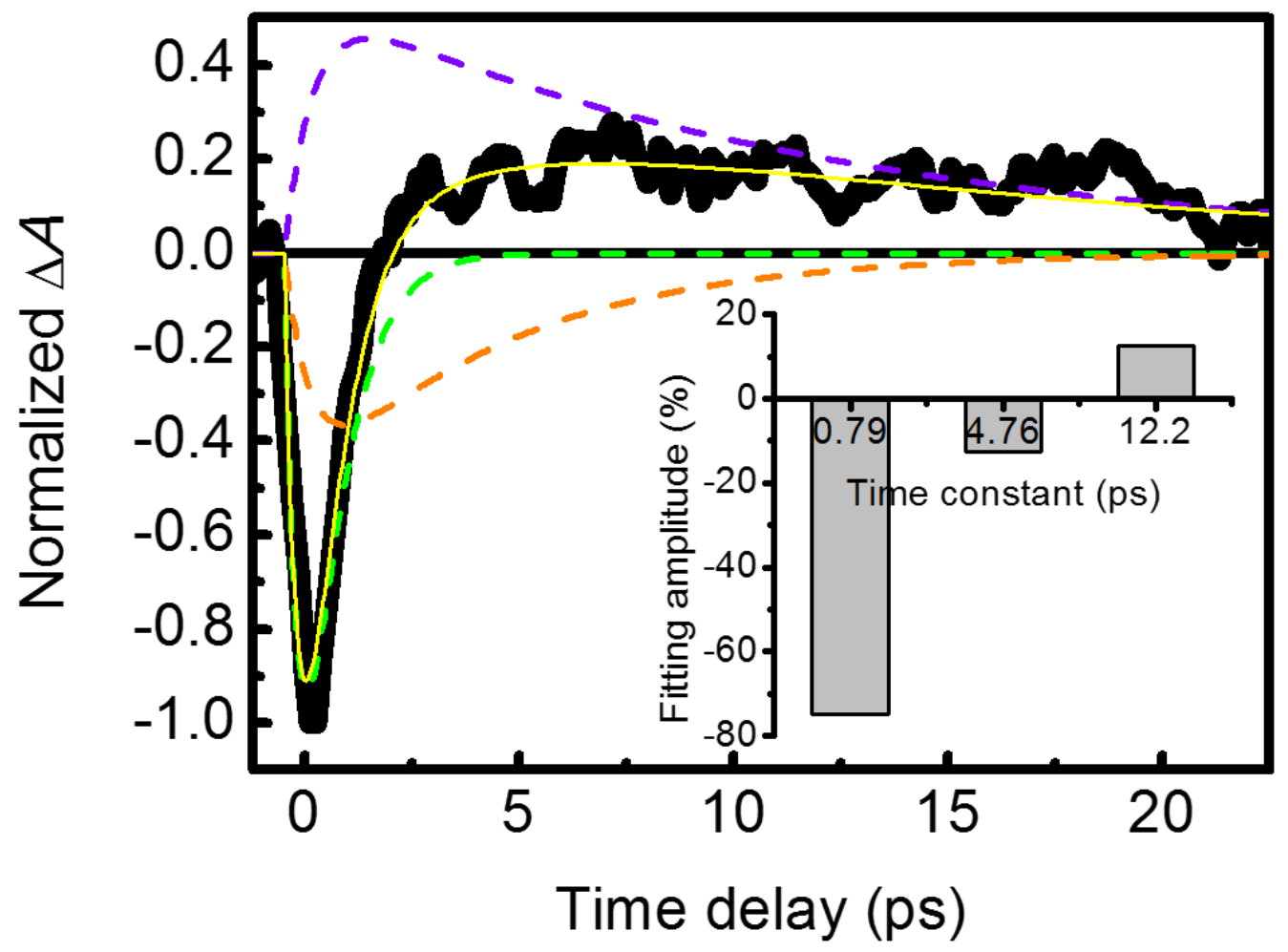


Table 1.

Fitting parameters

\begin{tabular}{ccccccccc}
\hline Sample & $\begin{array}{c}\mathrm{A}_{1} \\
(\%)\end{array}$ & $\begin{array}{c}\tau_{1} \\
(\mathrm{ps})\end{array}$ & $\begin{array}{c}\mathrm{A}_{2} \\
(\%)\end{array}$ & $\begin{array}{c}\tau_{2} \\
(\mathrm{ps})\end{array}$ & $\begin{array}{c}\mathrm{A}_{3} \\
(\%)\end{array}$ & $\begin{array}{c}\tau_{3} \\
(\mathrm{ps})\end{array}$ & $\begin{array}{c}\mathrm{A}_{4} \\
(\%)\end{array}$ & $\begin{array}{c}\tau_{4} \\
(\mathrm{ps})\end{array}$ \\
\hline eNOS-oxy/Im & -76.5 & 0.31 & - & - & 18.3 & 1.16 & 5.2 & 144 \\
eNOS-oxy/L-Arg & -47.6 & 0.36 & -52.4 & 1.4 & - & - & - & - \\
eNOS-oxy & -74.8 & 0.79 & -12.4 & 4.76 & 15.3 & 12.2 & - & - \\
\hline
\end{tabular}

Fitting parameters of $\Delta A$ traces of each sample at probing wavelength $405 \mathrm{~nm}$ 


\section{Reference}

${ }^{1}$ Moncada, S.; Higgs, E. A. The Discovery of Nitric Oxide and Its Role in Vascular Biology. Br. J. Pharmacol. 2006, 147 (S1), S193-S201. DOI: http://dx.doi.org/10.1038/sj.bjp.0706458

${ }^{2}$ Schmidt, H. H. H. W.; Walter, U. NO at Work. Cell 1994, 78 (6), 919-925. DOI: http://dx.doi.org/10.1016/0092-8674(94)90267-4

${ }^{3}$ Gautier, C.; Mikula, I.; Nioche, P.; Martasek, P.; Raman, C. S.; Slama-Schwok, A. Dynamics of NO Rebinding to the Heme Domain of NO Synthase-like Proteins from Bacterial Pathogens. Nitric Oxide Biol. Chem. 2006, 15 (4), 312-327. DOI: http://dx.doi.org/10.1016/j.niox.2006.03.010

${ }^{4}$ Montague, P. R.; Gancayco, C. D.; Winn, M. J.; Marchase, R. B.; Friedlander, M. J. Role of NO Production in NMDA Receptor-Mediated Neurotransmitter Release in Cerebral Cortex. Sci. 1994, 263 (5149), 973-977. DOI: http://dx.doi.org/10.1126/science.7508638

5 Terenzi, F.; Diaz-Guerra, M. J. M.; Casado, M.; Hortelano, S.; Leoni, S.; Bosca, L. Bacterial Lipopeptides Induce Nitric Oxide Synthase and Promote Apoptosis through Nitric Oxide-Independent Pathways in Rat Macrophages. Journal of Biological Chemistry, 1995, 270, 6017-6021. DOI: http://dx.doi.org/10.1074/jbc.270.11.6017

${ }^{6}$ Pollock, J. S.; Förstermann, U.; Mitchell, J. A.; Warner, T. D.; Schmidt, H. H.; Nakane, M.; Murad, F. Purification and Characterization of Particulate Endothelium-Derived Relaxing Factor Synthase from Cultured and Native Bovine Aortic Endothelial Cells. Proc. Natl. Acad. Sci. 1991, 88 (23), 1048010484. DOI: http://dx.doi.org/10.1073/pnas.88.23.10480

7 Stuehr, D. J.; Kwon, N. S.; Nathan, C. F.; Griffith, O. W.; Feldman, P. L.; Wiseman, J. N Omega-Hydroxy-L-arginine Is an Intermediate in the Biosynthesis of Nitric Oxide from ${ }_{L}$-arginine. J. Biol. Chem. 1991, 266 (10), 6259-6263.

${ }^{8}$ Hevel, J. M.; White, K. A.; Marletta, M. A. Purification of the Inducible Murine Macrophage Nitric Oxide Synthase. Identification as a Flavoprotein. J. Biol. Chem. 1991, 266 (34), 22789-22791.

9 McMillan, K.; Bredt, D. S.; Hirsch, D. J.; Snyder, S. H.; Clark, J. E.; Masters, B. S. Cloned, Expressed Rat Cerebellar Nitric Oxide Synthase Contains Stoichiometric Amounts of Heme, Which Binds Carbon Monoxide. Proc. Natl. Acad. Sci. 1992, 89 (23), 11141-11145.

${ }^{10}$ Stuehr, D. J.; Ikeda-Saito, M. Spectral Characterization of Brain and Macrophage Nitric Oxide Synthases. Cytochrome P-450-like Hemeproteins That Contain a Flavin Semiquinone Radical. J. Biol. Chem. 1992, 267 (29), 20547-20550.

11 Narhi, L. O.; Fulco, A. J. Identification and Characterization of Two Functional Domains in Cytochrome P-450BM-3, a Catalytically Self-Sufficient Monooxygenase Induced by Barbiturates in Bacillus Megaterium. J. Biol. Chem. 1987, 262 (14), 6683-6690. 
12 Chen, P. F.; Tsai, A. L.; Wu, K. K. Cysteine 184 of Endothelial Nitric Oxide Synthase Is Involved in Heme Coordination and Catalytic Activity. J. Biol. Chem. 1994, 269 (40), 25062-25066.

13 Nishimura, J. S.; Martasek, P.; Mcmillan, K.; Salerno, J. C.; Liu, Q.; Gross, S. S.; Masters, B. S. S. Modular Structure of Neuronal Nitric Oxide Synthase: Localization of the Arginine Binding Site and Modulation by Pterin. Biochem. Biophys. Res. Commun. 1995, 210 (2), 288-294. DOI:

http://dx.doi.org/10.1006/bbrc.1995.1659

14 Xie, Q. W.; Cho, H. J.; Calaycay, J.; Mumford, R. A.; Swiderek, K. M.; Lee, T. D.; Ding, A.; Troso, T.; Nathan, C. Cloning and Characterization of Inducible Nitric Oxide Synthase from Mouse Macrophages. Sci. 1992, 256 (5054), 225-228. DOI: http://dx.doi.org/10.1126/science.1373522 15 Abu-Soud, H. M.; Stuehr, D. J. Nitric Oxide Synthases Reveal a Role for Calmodulin in Controlling Electron Transfer. Proc. Natl. Acad. Sci. U. S. A. 1993, 90 (22), 10769-10772. DOI: http://dx.doi.org/10.1073/pnas.90.22.10769 16 Chen, P.; Tsai, A.; Berka, V.; Wu, K. K. Evidence for Bidomain Structure and Successful Reconstitution of Catalytic Activity. Biochemistry 1996, 271 (24), 14631-14635. DOI: http://dx.doi.org/10.1074/jbc.271.24.14631

17 Gerber, N. C. Endothelial Nitric-Oxide Synthase. J. Biol. Chem. 1996, 271 (19), 11462-11467. DOI: http://dx.doi.org/10.1074/jbc.271.19.11462

18 Rousseau, D. L.; Li, D.; Couture, M.; Yeh, S. R. Ligand-Protein Interactions in Nitric Oxide Synthase. J. Inorg. Biochem. 2005, 99 (1), 306-332. DOI: http://dx.doi.org/10.1016/j.jinorgbio.2004.11.007 19 Feng, C.; Thomas, C.; Holliday, M. a; Tollin, G.; Salerno, J. C.; Ghosh, D. K.; Enemark, J. H.; Carolina, N.; York, N. Direct Measurement by Laser Flash Photolysis of Intramolecular Electron Transfer in a Two-Domain Construct of Murine Inducible Nitric Oxide Synthase. J. Am. Chem. Soc. 2006, 128 (11), 3808-3811. DOI: http://dx.doi.org/10.1021/ja0578606

20 Feng, C. Mechanism of Nitric Oxide Synthase Regulation: Electron Transfer and Interdomain Interactions. Coord. Chem. Rev. 2012, 256 (3-4), 393-411. DOI: http://dx.doi.org/10.1016/j.ccr.2011.10.011

21 Belliston-Bittner, W.; Dunn, A. R.; Nguyen, Y. H. Le; Stuehr, D. J.; Winkler, J. R.; Gray, H. B. Picosecond Photoreduction of Inducible Nitric Oxide Synthase by rhenium(I)-Diimine Wires. J. Am. Chem. Soc. 2005, 127 (45), 15907-15915. DOI: http://dx.doi.org/10.1021/ja0543088 ${ }^{22}$ Mikula, I.; Durocher, S.; Martasek, P.; Mutus, B.; Slama-Schwok, A. Isoform-Specific Differences in the Nitrite Reductase Activity of Nitric Oxide Synthases under Hypoxia. Biochem. J. 2009, 418 (3), 673-682. DOI: http://dx.doi.org/10.1042/BJ20080987

${ }^{23}$ Li, D.; Stuehr, D. J.; Yeh, S.-R.; Rousseau, D. L. Heme Distortion Modulated by Ligand-Protein Interactions in Inducible Nitric-Oxide Synthase. J. Biol. Chem. 2004, 279 (25), 26489-26499. DOI: 
http://dx.doi.org/10.1074/jbc.M400968200

${ }^{24}$ McMillan, K.; Masters, B. S. S. Optical Difference Spectrophotometry as a Probe of Rat Brain Nitric Oxide Synthase Heme-Substrate Interaction. Biochemistry 1993, 32 (38), 9875-9880. DOI: http://dx.doi.org/10.1021/bi00089a001

25 Chen, P. F.; Tsai, A. L.; Berka, V.; Wu, K. K. Mutation of Glu-361 in Human Endothelial Nitric-Oxide Synthase Selectively Abolishes L-arginine Binding without Perturbing the Behavior of Heme and Other Redox Centers. J. Biol. Chem. 1997, 272 (10), 6114-6118. DOI: http://dx.doi.org/10.1074/jbc.272.10.6114

${ }^{26}$ Maes, E. M.; Roberts, S. a.; Weichsel, A.; Montfort, W. R. Ultrahigh Resolution Structures of Nitrophorin 4: Heme Distortion in Ferrous CO and NO Complexes. Biochemistry 2005, 44 (38), 1269012699. DOI: http://dx.doi.org/10.1021/bi0506573

27 Huszánk, R.; Lendvay, G.; Horváth, O. Air-Stable, Heme-like Water-Soluble Iron(II) Porphyrin: In Situ Preparation and Characterization. J. Biol. Inorg. Chem. 2007, 12 (5), DOI: http://dx.doi.org/681690. 10.1007/s00775-007-0217-y

${ }^{28}$ Negrerie, M.; Cianetti, S.; Vos, M. H.; Martin, J. L.; Kruglik, S. G. Ultrafast Heme Dynamics in Ferrous versus Ferric Cytochrome c Studied by Time-Resolved Resonance Raman and Transient Absorption Spectroscopy. J. Phys. Chem. B, 2006, 110 (25), 12766-12781. DOI: http://dx.doi.org/10.1021/jp0559377

${ }^{29}$ Wang, W.; Ye, X.; Demidov, A. A.; Rosca, F.; Sjodin, T.; Cao, W.; Sheeran, M.; Champion, P. M. Femtosecond Multicolor Pump-Probe Spectroscopy of Ferrous Cytochrome c. J. Phys. Chem. B, 2000, 104 (46), 10789-10801. DOI: http://dx.doi.org/10.1021/jp0008602

30 Chen, Z.; Jeffrey, A. S.; Justin, J. L.; Lijun, G.; Lijuan, W.; and Dongping, Z. Ultrafast Proteinquake Dynamics in Cytochrome c. J. Am. Chem. Soc., 2009, 131 (8), 2846-2852. DOI: http://dx.doi.org/10.1021/ja8057293

31 Marques, H. M.; Brown, K. L. Molecular Mechanics and Molecular Dynamics Simulations of Porphyrins, Metalloporphyrins, Heme Proteins and Cobalt Corrinoids. Coord. Chem. Rev. 2002, 225 (1-2), 123-158. DOI: http://dx.doi.org/10.1016/S0010-8545(01)00411-8

32 Ansari, A.; Berendzen, J.; Bowne, S. F.; Frauenfelder, H.; Iben, I. E. T.; Sauke, T. B.; Shyamsunder, E.; Young, R. D. Protein states and proteinquakes. Proc. Natl. Acad. Sci. U.S.A. 1985, 82 (15), 5000-5004. DOI: http://dx.doi.org/10.1073/pnas.82.15.5000

33 Dadusc, G.; Ogilvie, J. P.; Schulenberg, P.; Marvet, U.; Miller, R. J. D.; Diffractive optics-based heterodyne-detected four-wave mixing signals of protein motion: From "protein quakes" to ligand escape for myoglobin. Proc. Natl. Acad. Sci. U.S.A. 2001, 98 (11), 6110-6115. DOI: 
http://dx.doi.org/10.1073/pnas.101130298

34 Marten H. Vos.; Ultrafast dynamics of ligands within heme proteins. Biochimica et Biophysica Acta. 2008, 1777 (1) 15-31.DOI: http://dx.doi.org/10.1016/j.bbabio.2007.10.004

35 Franzen, S.; Wallace-Williams, S. E.; Shreve, A. P. Heme Charge-Transfer Band III Is Vibronically Coupled to the Soret Band. J. Am. Chem. Soc. 2002, 124 (24), 7146-7155. DOI: 10.1021/ja0172722

36 Franzen S.; Kiger L.; Poyart C.; Martin JL.; Heme photolysis occurs by ultrafast excited state metal-to-ring charge transfer. Biophys J. 2001, 80 (5), 2372-2385. DOI: http://dx.doi.org/10.1016/S0006-3495(01)76207-8

37 U. Liebl, L. Bouzhir-Sima, M. Négrerie, J.-L. Martin, M.H. Vos,; Ultrafast ligand rebinding in the heme domain of the oxygen sensors FixL and Dos: general regulatory implications for heme-based sensors. Proc. Natl. Acad. Sci. U. S. A. 2002, 99 (20), 12771-12776. DOI: http://dx.doi.org/10.1073/pnas.192311699

${ }^{38}$ Kurokawa, H.; Lee, D. S.; Watanabe, M.; Sagami, I.; Mikami, B.; Raman, C. S.; Shimizu, T. A Redox-Controlled Molecular Switch Revealed by the Crystal Structure of a Bacterial Heme PAS Sensor. J. Biol. Chem. 2004, 279 (19), 20186-20193. DOI: http://dx.doi.org/10.1074/jbc.M314199200

39 Park, H.; Suquet, C.; Satterlee, J. D.; Kang, C. Insights into Signal Transduction Involving PAS Domain Oxygen-Sensing Heme Proteins from the X-Ray Crystal Structure of Escherichia Coli Dos Heme Domain (Ec DosH). Biochemistry 2004, 43 (10), 2738-2746. DOI: http://dx.doi.org/10.1021/bi035980p

${ }^{40}$ Lim, M.; Jackson, T. a.; Anfinrud, P. a. Femtosecond Near-IR Absorbance Study of Photoexcited Myoglobin: Dynamics of Electronic and Thermal Relaxation. J. Phys. Chem. 1996, 100 (29), 1204312051. DOI: http://dx.doi.org/10.1021/jp9536458 